\title{
Improved Lagrangian relaxation generation decision-support in presence of electric vehicles
}

\author{
Hossein Zeynal ${ }^{1}$, Zuhaina Zakaria ${ }^{2}$, Ahmad Kor ${ }^{3}$ \\ ${ }^{1}$ Department of Electrical Engineering, Tehran Farayand Dena Engineering Co., Tehran, Iran \\ ${ }^{2}$ Faculty of Electrical Engineering, Universiti Teknologi MARA, 40450 Shah Alam, Selangor, Malaysia \\ ${ }^{3}$ Electrical Design Department, Kharazm Power Engineers Co., Tehran, Iran
}

\begin{tabular}{l} 
Article Info \\
\hline Article history: \\
Received Jul 14, 2020 \\
Revised Mar 9, 2021 \\
Accepted Mar 25, 2021 \\
\hline Keywords: \\
Cuckoo search algorithm \\
Duality gap \\
Electric vehicle \\
Generation scheduling \\
Unit commitment
\end{tabular}

Article Info

icle history:

Received Jul 14, 2020

Revised Mar 9, 2021

Accepted Mar 25, 2021

Duality gap

Generation scheduling

\begin{abstract}
Decision making strategies for resources available in macro/micro scales have long been a critical argument. Among existing methods to address such a mixed-binary optimization model, Lagrangian relaxation (LR) found universal acceptance by many utilities, offering a fast and accurate answer. This paper aims at retrofitting the solution way of LR algorithm by dint of meta-heuristic cuckoo search algorithm (CSA). When integrating CSA into LR mechanism, a tighter duality gap is catered, representing more accurate feasible solution. The key performance of CSA exhibits a head start over other classical methods such as gradient search (GS) and Newton Raphson (NR) when dealt with the relative duality gap closure in LR procedure. Further, electric vehicles (EV) with its associated hard constraints are encompassed into model to imperiling the proposed CSA-LR if encountered with nonlinear fluctuation of duality gap. Simulation results show that the proposed CSA-LR model outperforms the solution quality with/without EV as compared with conventional NR-LR method.
\end{abstract}

This is an open access article under the CC BY-SA license.

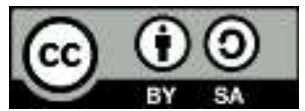

\section{Corresponding Author:}

Hossein Zeynal

Department of Electrical and Computer Engineering

Buein Zahra Technical University

Buein Zahra, Qazvin, Iran

Email: hzeynal@bzte.ac.ir

\section{INTRODUCTION}

An electric power system is consisting of a big number of generating units. Enough number of units must be committed (switched-ON) to meet the electricity demand. In this sense, finding the best set (cost-effective) of generating units to start-up is known as unit commitment (UC) process. As a result, UC builds an optimization problem whose objective is to minimize production costs for all generating units subject to a number of temporal and operational constraints for each individual generation unit and in couple with other units [1-7].

Smart grid development in all levels of generation, transmission and distribution, has resulted in a complex optimal operation of the system. For instance, large arrival of electric vehicle (EV) into the transportation system complicates economic operation of the power system [8]. Such dynamic vehicle introduces dual operational modes of charging and discharging. However, it offers a dichotomy in optimal operation of the system. It is adventurous if EVs can be scheduled to connect and disconnect to the power grid in suitable time and optimal place to improve the security of the power grids [9-12].

As a result, the old-fashioned and conventional UC algorithm as a start-up scheduler can be retrofitted to address new challenges that recently raised by EVs. UC may coordinate geographically located 
thermal units with dynamic EVs to reconcile electricity demand in premise of satisfying system security requirements. To further flourish the UC solution, the model can be enhanced by adding PV/battery generations into the final schedule. In this fashion, UC redresses the schedule for conventional units, EVs, and PV/battery in a cost-saving and environmental-friendly way [9].

To solve the resultant UC optimization problem, a variety of techniques reported in technical literature. However, in such pool of possible solutions, few have received the industry approval on large-scale UC implementation. Nevertheless, the most appreciated techniques include priority listing (PL), dynamic programming (DP), Lagrangian relaxation (LR), and mixed integer programming (MIP) [13-20].

In the proposed project, LR technique is employed to solve the resultant UC problem. According to the literature survey, LR outperforms other techniques in terms of computing time and linear solution as the size of the problem enlarges [7, 17, 18, 21-23]. Although the LR presents many industrially-precious advantages, it still suffers from heuristic reasoning (try and error process) existed in tightening dual gap in LR convergence. Another major obstacle into broad application of LR is the incapability of LR to handle coupling constraints in an efficient way (time and cost overrun and unnecessary commitment of the units) [3, 17-19]. The proposed paper enhances UC solution using LR method while the duality gap fluctuation is optimized using biologically-inspired cuckoo search algorithm (CSA). In this sense, CSA finds the best update for the Lagrangian multiplier at each iteration. As a consequence, the executional performance of the UC-LR can be improved and unnecessary commitment of the units is avoided [6-8, 15-22].

It is widely proven that the UC is the most money-saving procedure in power system operation. In fact, UC sets a pre-dispatch start-up schedule for the in-service units in the system [10, 11]. However, it builds up a large-scale, nonlinear, non-differentiable, nonconvex mixed-integer mathematical programming problem with a complex constraint set $[6,7,14,15]$. Having discrete decision making process that involves with a big number of binary variables may exacerbate the solution process or in some cases unable to reach the most optimal solution. Therefore, the conventional optimization techniques used to solve the economic dispatch problem is no longer fruitful. A number of solution approaches are proposed in the literature. Amongst them, the most industry-proven methods are dynamic programming (DP), Lagrangian relaxation (LR) and mixed-integer programming (MIP) methods [3, 4, 9, 18].

Lagrangian relaxation (LR) decomposes the UC problem into several subproblems [3], whereas the solution of each subproblem is coordinated through Lagrange multipliers. The subproblems are mainly solved by the DP or a network flow technique. Mixed-integer programming (MIP) technique using branch and cut $(\mathrm{B} \& \mathrm{C})$ method is applied to the UC problem as well [3].

While DP is notorious for dimensionality curse, LR is well-known for linear computational performance that tremendous scale outruns the DP method. Nevertheless, MIP outperforms the DP and LR techniques by enabling direct modeling of the coupling constraints which proved cumbersome in LR model. Although MIP may offer better optimal solution but it is still based on approximation and linearization of the initial model. Compared to LR, MIP is unable to model non-linear objective and constraints. By having it converted into equivalent linear model will be at the expense of deteriorating feasibility [2, 4]. MIP is a recent in-favor solution as it utilizes a super-optimizer of ILOG IBM CPLEX that uses branch and bound with Cutting plane method (BB\&C) [4-10].

Comparison over these industry-size techniques has manifested that the LR technique still offers satisfactory solution (high-speed) although suffering from large fluctuation of duality gap and its uncontrollability at the premise of system-wise constraints. As a remedy, stochastic search algorithm such as genetic algorithm (GA), particle swarm optimization (PSO) can provide stochastic way of non-linear optimization which is inspired by biologically advancement in the nature. The advantages of such techniques was cited in many technical literatures especially for complex mathematical equations for a nonlinear problem is a formidable task $[15,16,19-23]$.

Among several meta-heuristic algorithms, cuckoo search algorithm (CSA) recently emerged and suggests straightforward and user-friendly implementation mechanism as opposed to many well-known algorithms. Thus, integration of CSA into LR solution paradigm ought to improve the overall performance both in computational sense of large-scale problems with solution optimality and minimization of operation costs [20-27].

The objective of this paper is to refine better quality solution of LR with the stochastic search engine of CSA. To end this, CSA replaces NR engines in LR algorithm which seemingly presented an inferior performance in utility-size number of units. To examine the strengths of the proposed model, electric vehicle (EV) operational constraints are added into the legacy model. The resultant model will be tested on New-England 39-bus (10-unit) system to show the efficacy of the proposed model. 


\section{MATHEMATICAL EQUATIONS}

The generic form of LR model is illustrated in (1-3). The objective is to minimizing total cost function, $f(x)$, subject to a set of equality $g_{i}(x)$ and inequality $h_{i}(x)$ constraints [2,3].

$$
\min _{x} f(x)
$$

subject to

$$
\begin{aligned}
& g_{i}(x)=0 \quad \forall i=1, \ldots, m \\
& h_{i}(x) \leq 0 \quad \forall i=1, \ldots, m \\
& x \in X
\end{aligned}
$$

The LR model solve UC problem by temporarily relaxing coupling constraints and adds them up into the objective matrix by using dual optimization procedure. It can readily be done through the adjustment of Lagrange multiplier (LM), denoted as $\lambda$ to obtain constrained optimum. The (4) represents the Lagrangian function in respect with the approximation of original problem (1-3). Lagrangian multipliers are ought to penalize violations of decoupling constraints in primal solution by adding extra cost on the objective value, which is also the dual solution [3].

$$
\begin{aligned}
& \Phi(\lambda, \mu)=\min _{x}\left\{f(x)+\lambda^{T} g(x)+\mu^{T} h(x)\right\} \\
& \text { subject to } x \in X
\end{aligned}
$$

$\Phi(\lambda, \mu)$ is defined as a dual cost function. To yield a lower bound primal problem feasible solution, one should maximize $\Phi$ with respect to $\lambda$ and $\mu$ as represented in (4).

$$
\begin{aligned}
& q^{*}(\lambda)=\max _{\lambda, \mu} \Phi(\lambda, \mu) \\
& \text { subject to } \mu \geq 0
\end{aligned}
$$

The lagrange relaxation procedure solves the unit commitment problem by a duality gap in (21). The $\mathrm{J}^{*}$ is obtained by performing economic dispatch on committed units to procure optimal generation output in terms of cost. The parameter $\mathrm{q}^{*}$ is obtained by calculating the minimum lagrange function as shown in (22) $\&(23)[2]$.

$$
\mathrm{RDG}=\frac{J^{*}-q^{*}}{q^{*}}
$$

The status of generation units can be determined using (1-5) where single-unit dynamic programming approach is taken up finding commitment ( $0 / 1$ status) of each unit throughout scheduling horizons, eg. 24 hours.

The relative duality gap (RDG) as stated in (6) plays a crucial role in LR algorithm, it controls the accuracy of the solution by closing the gap between primal and dual problem solutions as in (6). Following the LR solution mechanism, the Lagrange multiplier (LM), $\lambda$, required updating each iteration using (7) and with fresh LM the algorithm repeats till the pre-specified RDG is met. The $\alpha$ parameter accelerates convergence speed while closing the relative duality gap between two-end solutions [3].

$$
\lambda^{t+1}=\lambda^{t}+\left[\frac{d}{d \lambda} q(\lambda)\right] \alpha
$$

\subsection{Objective function}

The generation scheduling problem using LR algorithm was stated in a general form. The resultant problem is a large-scale, mixed-integer, non-linear, non-convex, hard optimization problem. Detailed problem model in terms of objective function and its associated constraints can be cast as [2-10]:

$$
\underset{P_{i}, I_{i}}{\min } \mathrm{F}(\mathrm{x})=\left\{\sum_{t=1}^{N T} \sum_{i=1}^{N G}\left(a P_{i}^{2}(t)+b P_{i}(t)+c\right) I_{i}(t)+\sum_{t}^{N T} \sum_{i}^{N G} S U_{i}(t) . I_{i}(t)\right\}
$$

The power-balance constraint: 


$$
\sum_{i}^{N G} P_{i}(t) . I_{i}(t)+P_{\text {loss }}+\sum_{v} P_{v}(t)=P_{d}(t)
$$

Spinning reserve constraint:

$$
\sum_{i=1}^{N G} \overline{P_{i}}(t) . I_{i}(t) \geq S R_{i}(t)
$$

Unit limit constraint:

$$
\underline{P_{i}}(t) . I_{i}(t) \leq P_{i}(t) \leq \overline{P_{i}}(t) . I_{i}(t)
$$

EV constraints:

$$
\begin{aligned}
& P_{v}(t)=P_{d c}(t)-P_{c}(t) \\
& I_{d c}(t)+I_{c}(t)=1 \\
& \underline{P_{d c}}(t) \cdot I_{d c}(t) \leq P_{d c}(t) \leq \overline{P_{d c}}(t) \cdot I_{d c}(t) \\
& \underline{P_{c}}(t) \cdot I_{c}(t) \leq P_{c}(t) \leq \overline{P_{c}}(t) \cdot I_{c}(t)
\end{aligned}
$$

Status:

$$
I_{i}(t), I_{c}(t), I_{d c}(t) \in\{0,1\}
$$

Mixed-binary Unit commitment problem based on LR approach which in fact embrace decoupling constraints is formulated in (8-16). The object is to minimize the total quadratic cost of power production including $a, b, c$ as the cost coefficients and the main variable which is $P_{i}(t)$ plus with start-up cost of the unit $S U_{i}(t)$ for all the generators $(\mathrm{NG})$ over the study period $(\mathrm{T})$ [1-5]. The production cost of a unit is approximated in a quadratic cost function with its coefficients.

The power balance equation is represented in (9) which alludes that the sum of all generation from thermal units, $P_{i}(t)$, and power injection/withdrawal from electric vehicles, $P_{v}(t)$, ought to be seamlessly equal to the total demand of the system, $P_{d}(t)$, considering transmission losses, $P_{\text {loss }}$, at each scheduling period. The power balance constraint in (9) is referred to as a hard and system-wise constraint which requires one to schedule all the generation units simultaneously in every time period. That is, the constraint is sometimes called a coupling constraint which engages all the unit in scheduling process. This however introduces a challenging task for LR algorithm to satisfy certain accuracy level while closing the RDG gap.

The constraint (10) ensures one that there will be enough spinning reserve, $S R_{i}(t)$, in the system when generations meet the loads to hold a certain reliability margin during operation. The generator's power output is controlled to fall in the capability range of the unit within its minimum and maximum deliverable power as in (11).

As a part of the prime intent of this paper, electric vehicle operations are incorporated into the UC model to further jeopardize LR solution mechanism when it comes to an increased number of coupling constraints stated in (12-15). The power delivered/withdrawn (+/-) to/from the grid is given in (12). As of the dual mode of EV fleets when connected to the grid, in (13) stresses that the charging and discharging operational mode of EV are mutually exclusive. The constraints (14) and (15) are account for charging and discharging limits of each EV fleet to be held when in service [8]. The binary variables, (16), of $I_{i}(t), I_{c}(t)$, $I_{d c}(t)$ represent the status of the thermal unit $(0 / 1)$ and charging and discharging of EV fleets in seek of minimized total cost of operations at each time interval as presented in (8).

\section{CUCKOO SEARCH ALGORITHM}

Cuckoo search (CS) is a metaheuristic search algorithm coined by Yang and Deb [25, 26]. Its intensification and diversification methods were formulated based on the fascinating brood parasitism of some species of cuckoos to solve both convex and non-convex problems. Following Figure 1 at which the overall procedure of LR-UC is depicted, as one can see in Figure 2 as well, Cuckoos lay their eggs in the nests of the other host birds and may destroy the hosts' eggs to increase the hatching probability of their own 
eggs. However, detection of alien eggs by the host birds will result in extruding the identified dissimilar egg from the nest or leaving the nest and construct fresh nest. In order to decrease the possibility of their eggs for being abandoned, some cuckoo species mimicry the color and pattern of the few chosen host species. Yet, some species of cuckoos are the temperate migrators where they inhabit wide range of habitat to maximize the potential brood host and resolve the food limitation from previous habitat. The detailed mathematical formulation is given in [24-27].

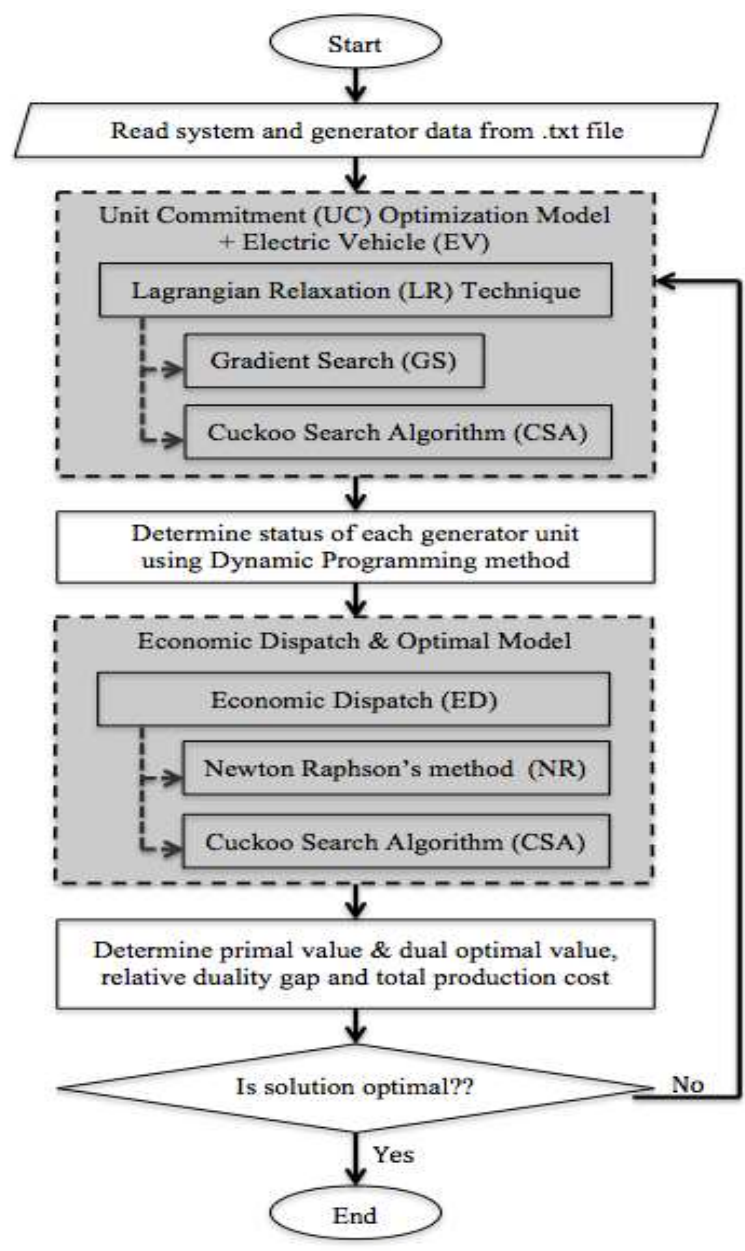

Figure 1. Flowchat of LR-UC-CSA

$$
\begin{aligned}
& X=\left[X_{1}, X_{2}, \ldots, X_{N P}\right]^{T} \\
& X_{i}=\left[P_{i 1}, P_{i 2}, \ldots, P_{i j}, \ldots, P_{i N}\right] \\
& P_{i j}=P_{j}^{\text {min }}+\operatorname{rand}_{1} *\left(P_{j}^{\text {max }}-P_{j}^{\text {min }}\right) \\
& X_{i}^{\text {new }}=\text { Xbest }_{i}+\alpha \times \operatorname{rand}_{2} \times \Delta X_{i}^{\text {new }}, \alpha>0 \\
& \Delta X_{i}^{\text {new }}=v \times \frac{\sigma_{x}(\beta)}{\sigma_{y}(\beta)} \times\left(X \text { best }_{i}-\text { Gbest }\right) \\
& v=\operatorname{rand}_{x} / \mid \text { rand }\left._{y}\right|^{1 / \beta} \\
& \sigma_{x}(\beta)=\left[\tau(1+\beta) \times \sin \left(\frac{\pi \beta}{2}\right) / \tau\left(\frac{1+\beta}{2}\right) \times \beta \times 2^{\frac{\beta-1}{2}}\right]^{1 / \beta}
\end{aligned}
$$




$$
\begin{aligned}
& \sigma_{y}(\beta)=1 \\
& 0.3 \leq \beta \leq 1.99 \\
& X_{i}^{\text {dis }}=\text { Xbest }_{i}+K \times \Delta X_{i}^{\text {dis }} \\
& \Delta X_{i}^{\text {dis }^{\text {dis }}}=\operatorname{rand}_{3} \times\left[\operatorname{randp}_{1}\left(\text { Xbest }_{i}\right)-\operatorname{randp}_{2}\left(\text { Xbest }_{i}\right)\right]
\end{aligned}
$$

In (17-20) represent the formulations for variables used in cuckoo search mechanism [24-27] and how they evolve using the Lévy flights via Mantegna algorithm is shown in (21-27) which attempts to estimate the best jump from the current position to approach the most optimal solution by adjusting the deviations of the next levy flight for cuckoos. Each egg in a nest represents a solution, and a cuckoo egg represents a new solution. The aim is to use the new and potentially better solutions (cuckoos) to replace an inferior solution in the nest. In this paper, each nest has one egg (solution). The algorithm can be extended to more complicated cases in which each nest has multiple eggs representing a set of solutions. The algorithm for the improved LR-UC tied with CSA is shown in Figure 1.

The prime aim is to find the best set of generator which can meet the load demand throughout the scheduling period at the premise of honoring system operational constraints. As can be seen in Figure 1, the CSA is integrated into the LR solution mechanism when the duality gap between the primal and dual solution are ought to be met. CSA nonlinearly finds a best path for gap evolution so that less fluctuation is seen as the LR-UC progresses [23]. Therefore, CSA challenge the process and offers an optimal way to curb the vaporized gap development. As a result, solution quality is enhanced.

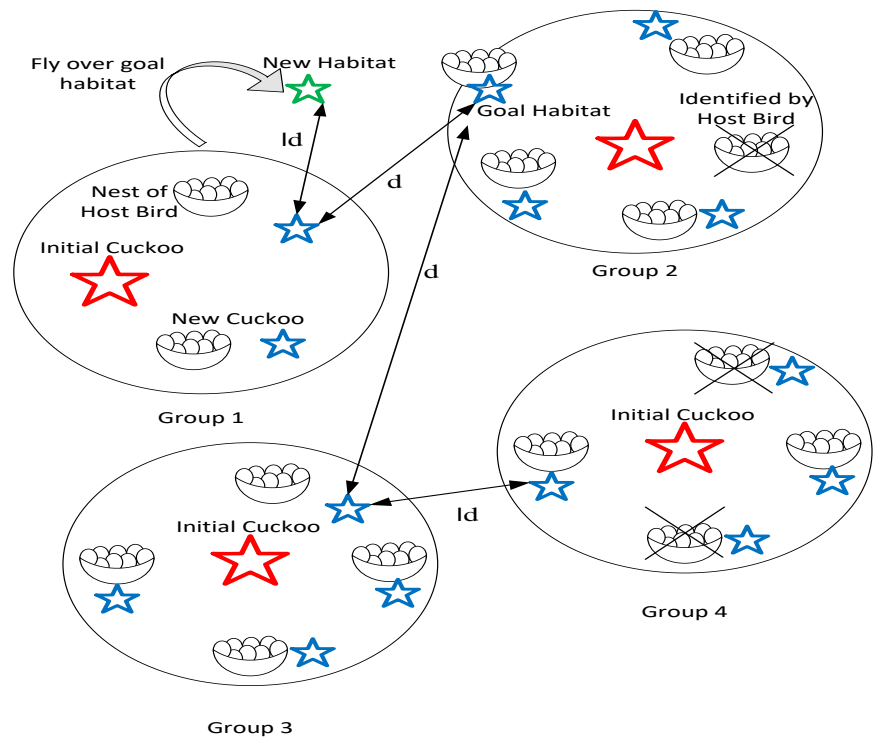

Figure 2. CSA graphical representation

\section{SIMULATION RESULTS}

In this section, two cases are analyzed to summarize the effectiveness of using CSA to solve UC-LR problem. The IEEE 39-bus test system is taken to examine the proposed CSA-UC-LR algorithm in an efficient way. IEEE 39-bus standard test system contains 49 buses, 32 transmission lines, 24 transformers and 10 generation unit which supplies Hourly power demand as given in Table 1. Studies are made on two cases as stated below. First case mainly focused on solving the feasibility solutions of LR in UC by integrating gradient search (GS) method and cuckoo search algorithm (CSA) respectively with and without electric vehicle $(\mathrm{EV})$.

On the other hand, second case concentrated on the optimal generation of units in ED using Newton Raphson's (NR) method and CSA. A total of ten thermal generators are run for both case in the time frame of 24-hours to testify the results. EV fleets are added into the system in a way that may represent its mobile charge/discharge capabilities. To end this, Figure 3 illustrates the time that EV fleets connects and discharge its power to the grid within hours 2-6 and charges back their batteries in hours of 8-11 and 20-22. Since the 
impact of network constraints has ignored in this model, therefore the driving pattern of EV fleets has no direct impact on the UC-LR solution considering EV fleets as a moveable unit. That is, the transmission network topology falls out of the scope of this paper. A total of $989 \mathrm{EVs}$ and $788 \mathrm{EVs}$ are participating in two discharging periods of hours 8-11 and 20-22 respectively along with 2000 EVs partaking in charging period of hours between $2-6$.

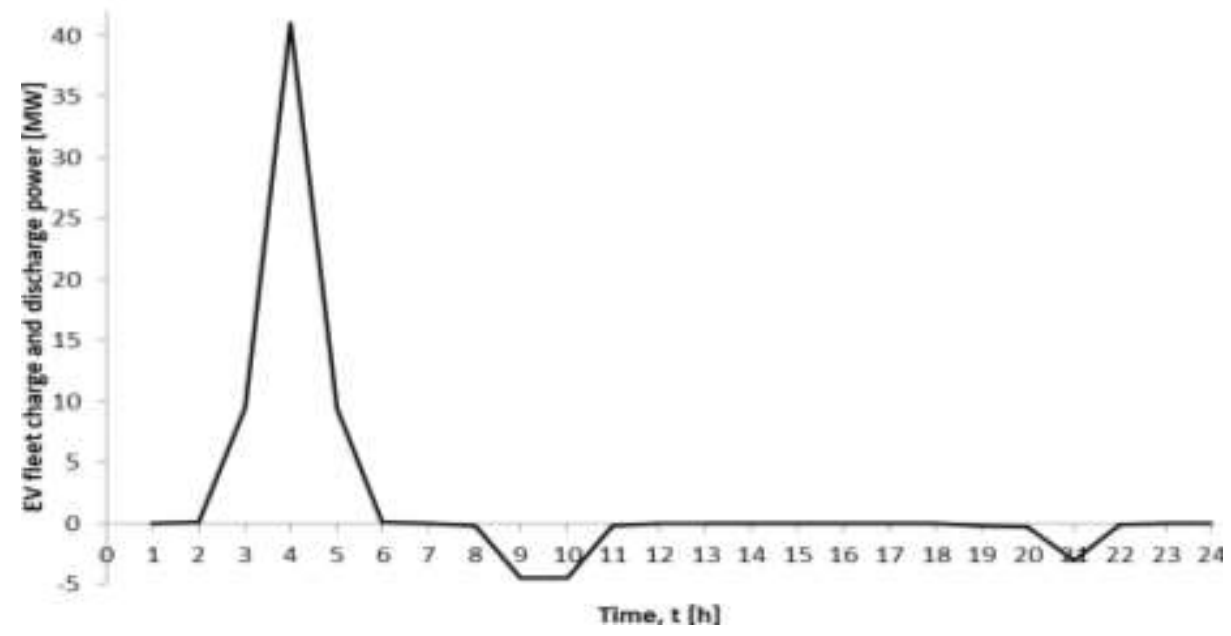

Figure 3. Comparison of different engines in UC-LR algorithm in presence of EV

As one can witness in Table 1, LR-CSA outperforms LR-GS in term of procuring precise LM as well as the smallest RDG value which indicates the closeness between dual and primal values. Another word, the lower RDG results in the near optimal solution is procured. Nevertheless, RDG amount is germane to LM value and by integration of GS into LR algorithm, it suffers from instable convergence at the end of the process. As can be seen in Table 1, some units keep switching their status (on=1 or off $=0$ ) repeatedly. Hence, the program executes for an infinite number of iterations till it manually becomes fixed as shown in Table 1 . In this table, the negative sign of RDG in LR-GS method means that the solution (total operation cost) was sub-optimized while the gap between dual and primal values being less than zero. It simply pinpoints that the cost is no longer minimized and in fact had deviated the preferred lowest cost.

Table 1. UC-LR solution in absent of EV fleets

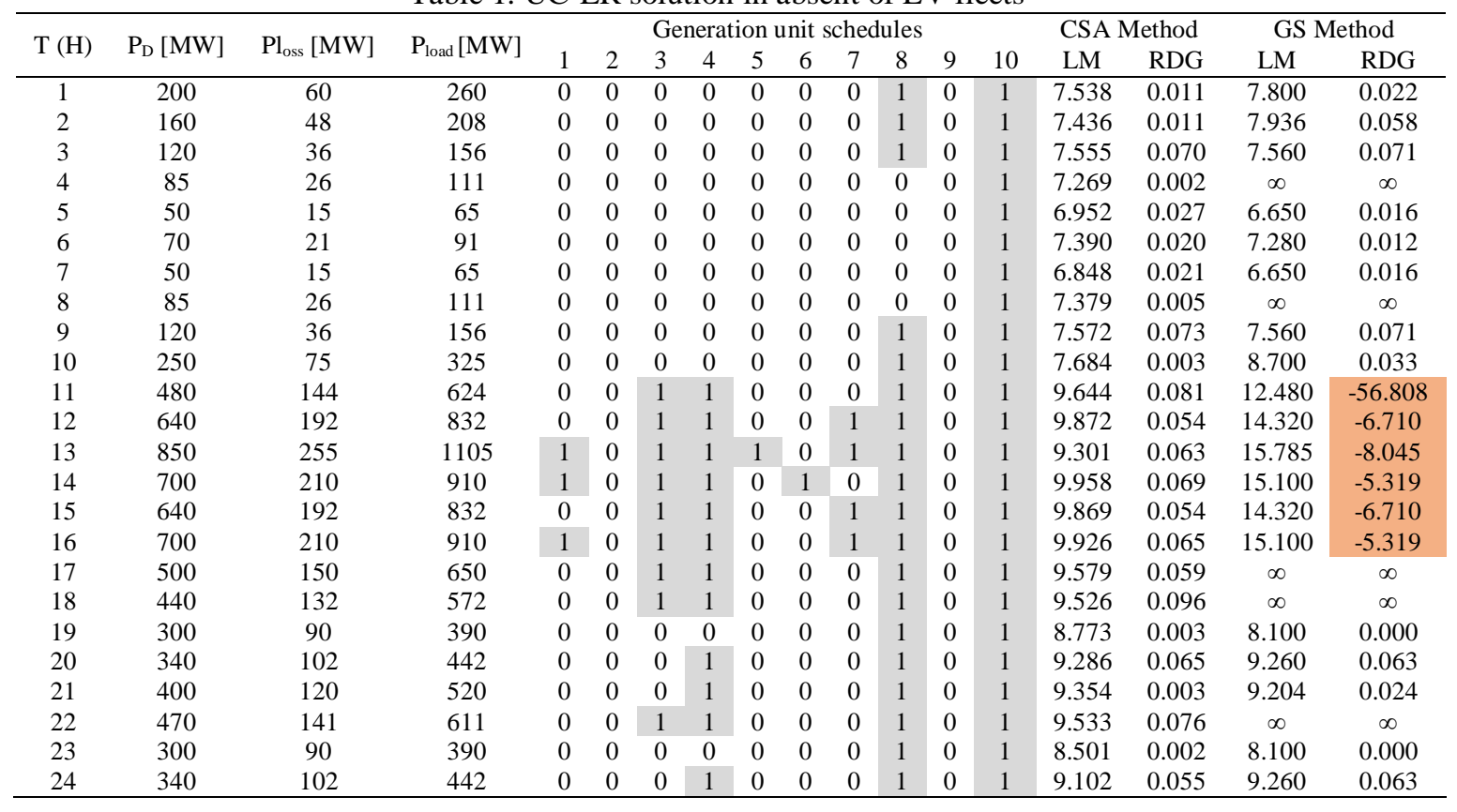


Case 1: Assuming 3\% fixed transmission loss in the system, where total hourly load somewhat fluctuates by arrival of the EV fleets connected to the grid. Tables 1 and 2 show the instability of LR-GS solution as the load is met at every time interval. Infeasible solutions represented by infinity loops are occurred as an extra number of generators is required to supply the increasing load. With advent of EV fleets, though this can be further worsened when plugged in or out the system such that, as shown in Figure 4, EV fleet is treated as a system demand (red color) in the forms of negative and positive load and vice versa.

As far as peak-load shaving is concerned, with arrival of EV fleets, the number of generators needed to meet the load at peak hours of operation can be reduced. Taking all into account the Tables 1 and 2 together with Figures 4 and 5, it is intuitively conceivable that LR-CSA offers not only better UC feasible solution with low-discrepancy LM and tightened RDG as compared to LR-GS but it provides one with affordable to solve probabilistic nature of EV fleets (charging and discharging states in accord to their State of charge (SOC)).

It is done through strong stochastic search engine existed in CSA solution mechanism that allows one to handle non-linear and random nature of the process. The capability of CSA in curbing the randomness of RDG closure efforts in LR technique has superbly made its broad application possible. It has long been argued that the LR-GS performs inferiorly and proves unreliable (infinity signs in Tables 1 and 2) as the number of coupling constraints increases, resulting in drastic deviations in RDG duality gap closing process. In this sense, LR-GS dwarfs its advantages when subject to randomness in the process. Conversely, CSA presents its strengths in terms handling non-linear and non-deterministic processes as the one expressed here.

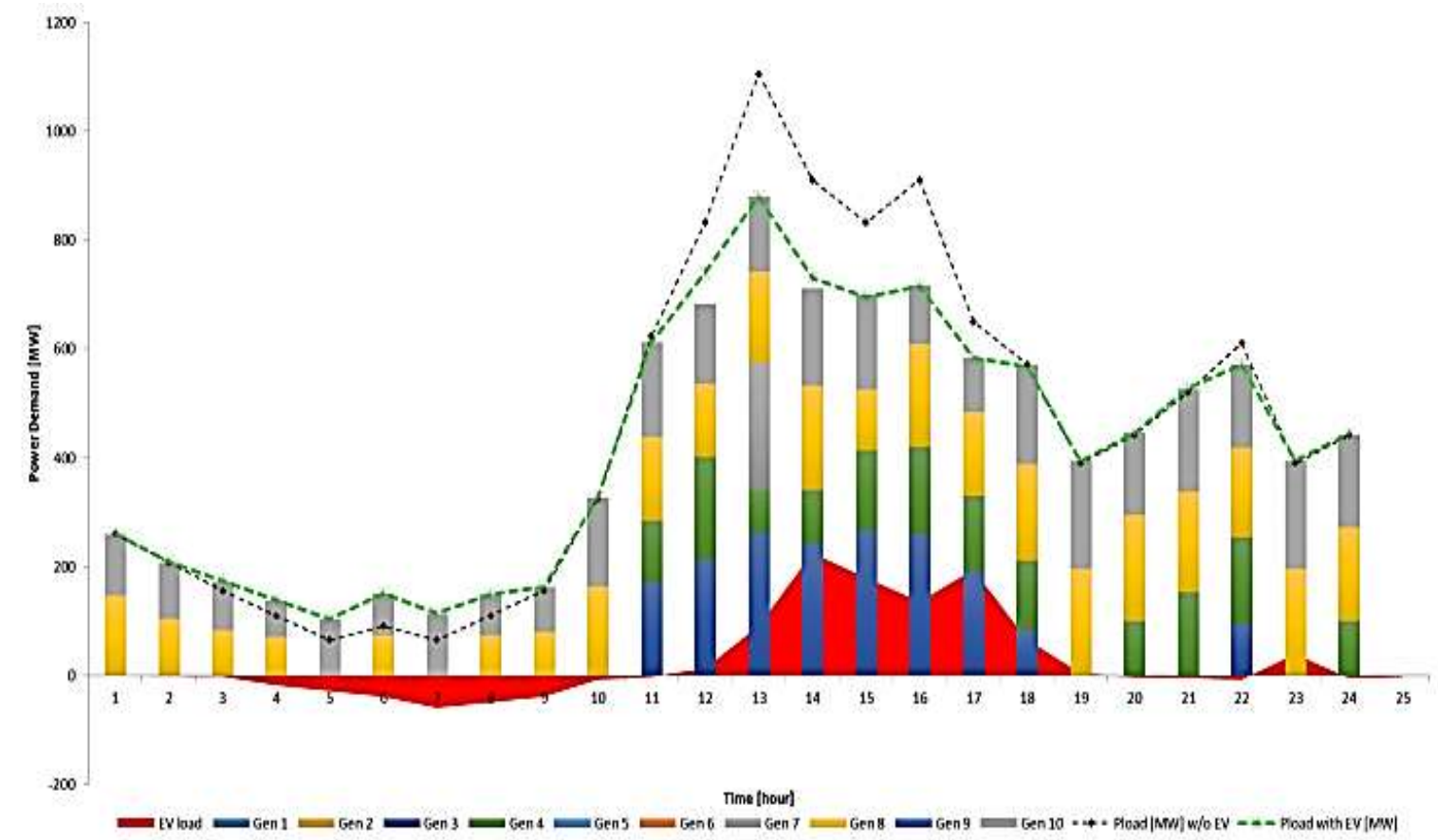

Figure 4. UC and ED solutions with and without EV

As a salient feature of CSA plugged in LR algorithm is when the searching process barely traps in the local optimum as opposed to GS mechanism which seemingly somewhat handicapped to grasp a global extreme point. As an added value, in terms of computational time performance of the LR-CSA, through Figure 5, it is demonstrable that, in spite of load variation in subsequent scheduling time-intervals, for 46 percent of the time, less than 13 seconds required to reach global final solution. However, it seems unattainable for LR-GS as it often locks up in local optima and it slowly trundles up to the global minima because it embraced a deterministic searching mechanism which relies on gradient vector (first order derivation) that is reluctant to deal with nonlinearity and randomness in the process. This, nonetheless, unequivocally reiterates the CSA procures concrete solution when integrated in LR algorithm. Instead, GS which merely outperforms CSA only when nonlinearity is infinitesimal and process possessed nondeterministic nature per se. 


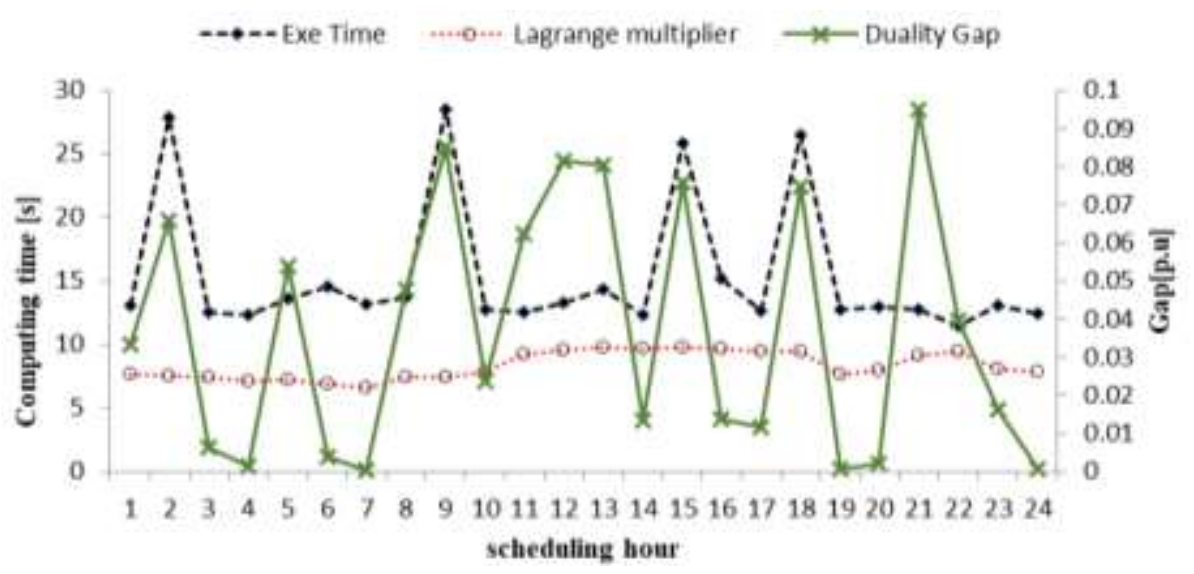

Figure 5. Comparative study for generation decision support

Table 2. The instability of LR-GS solution

\begin{tabular}{ccccc}
\hline & \multicolumn{4}{c}{ With EV } \\
T [h] & \multicolumn{2}{c}{ CSA } & \multicolumn{2}{c}{ GS } \\
\hline & LM & RDG & LM & RDG \\
\hline 1 & 8.062 & 0.0318 & 7.83 & 0.0192 \\
2 & 7.787 & 0.0388 & 7.978 & 0.0679 \\
3 & 7.643 & 0.0511 & 7.74 & 0.0656 \\
4 & 7.577 & 0.085 & $\infty$ & $\infty$ \\
5 & 7.331 & 0.0062 & 7.28 & 0.0042 \\
6 & 7.677 & 0.0877 & 7.5 & 0.0557 \\
7 & 7.345 & 0.0028 & $\infty$ & $\infty$ \\
8 & 7.65 & 0.0837 & 7.45 & 0.0559 \\
9 & 7.789 & 0.0895 & 7.63 & 0.0673 \\
10 & 7.975 & 0.0049 & 8.724 & 0.0325 \\
11 & 9.55 & 0.074 & $\infty$ & $\infty$ \\
12 & 9.541 & 0.0242 & $\infty$ & $\infty$ \\
13 & 9.893 & 0.0079 & $\infty$ & $\infty$ \\
14 & 9.701 & 0.0073 & $\infty$ & $\infty$ \\
15 & 9.661 & 0.0171 & $\infty$ & $\infty$ \\
16 & 9.684 & 0.0008 & $\infty$ & $\infty$ \\
17 & 9.53 & 0.0866 & $\infty$ & $\infty$ \\
18 & 9.545 & 0.0969 & $\infty$ & $\infty$ \\
19 & 8.154 & 0.0004 & 8.07 & 0.003 \\
20 & 9.144 & 0.0523 & 9.38 & 0.0687 \\
21 & 9.157 & 0.0149 & $\infty$ & $\infty$ \\
22 & 9.509 & 0.0913 & $\infty$ & $\infty$ \\
23 & 8.192 & 0.0004 & 8.06 & 0.0002 \\
24 & 9.241 & 0.0585 & 9.32 & 0.0648 \\
\hline & & & &
\end{tabular}

A detailed characteristic of LR-CSA method is illustrated in Figure 5. One can find that LM value is proportional to the load power as the demand increases from 10-16 hour, the Lagrange multiplier rise up roughly from 7 to $10 \$ / M W h$ and in the same trend as the load decreases LM falls in the range close to the 8 $\$ / M W h$. It should be noted that the rate of LM variation over load fluctuations is unsubstantial. Figure 5 further shows that value of duality gap drastically fluctuated on account of stochastic process performed by CSA. It is a process which is purely random with a heavy-tailed step size.

Case 2: LR-CSA is further investigated through comparison of the results with which LR-NR had offered based on the same test conditions (10-unit system added EV fleets). The result presented in Figure 6 taking into account EV fleet participation in the model.

According to Figure 6, The total production cost for LR-NR is found to be less than LR-CSA. The gulf between total cost of LR-CSA and its competitor LR-NR becomes wider especially during the peak loading periods in which additional generators required kicking on to meet the system's demand. During peak hours, it can be seen in Figure 6 that total production cost of $79650.78 \$$ and $81270.62 \$$ yielded from NR and CSA respectively.

From the simulation result, it turned out, the LR-NR is more cost-saving technique when solving ED sub-problem in UC model. It in fact, benefits from the approximation existed in NR mechanism, albeit it is 
yet unable to curb random deviations. It evidently might trap in local minima in some cases. Hence, although LR-NR outpaces LR-CSA in terms of production cost but it falls behind as the number of coupling constraints is growing in practical utilities which makes LR-NR undesirable choice in practice.

Furthermore, Figure 6 shows that 96 percent of time LR-NR provides cheaper solution than LR-CSA. The chances NR providing lower cost is 29 percent higher than when EV incorporated into the model. This represents that operational dynamism of EV fleets affects the LR-CSA method in finding optimal solution. Nevertheless, integration of EV reduced the total production cost in both methods in comparison to the cost without integration of EV.

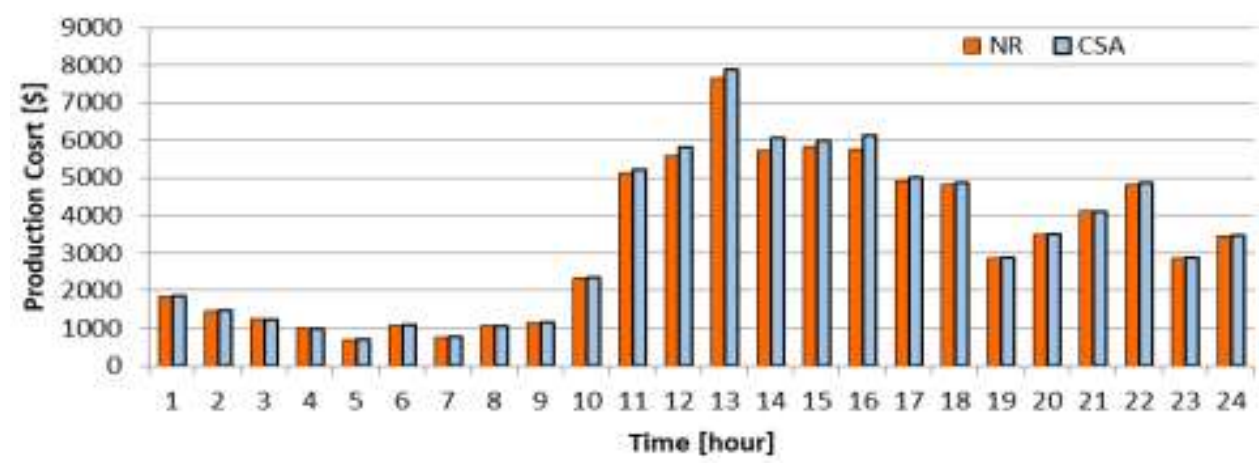

Figure 6. Production cost of LR-NR and LR-CSA methods

\section{CONCLUSION}

The unit commitment (UC) problem was solved by comparing gradient search (GS) method to cuckoo search algorithm (CSA) and Newton Raphson's (NR) method to CSA respectively. When integrating CSA into LR mechanism, a tighter duality gap ends up, representing more accurate feasible solution. LR-CSA improves Lagrange multipliers and relative duality gap by offering lower primal cost. With advant of electric vehicles (EV) into model, the proposed CSA-LR was further challenged by nonlinear fluctuation of duality gap. Simulation results LR-CSA outperforms traditional LR-GS method in terms of real-world utility-size with bigger number of generators. The numerical tests and results exhibit the fruitful solutions for UC problem with and without electric vehicle (EV). As a future work, a detailed EV charging model can be added to the model and if the model became more intractable, a recently evolved metahuristic method such as gray wolf optimization can be introduced to the solution process.

\section{REFERENCES}

[1] L. A. F. M. Ferreira, "On the duality gap for thermal unit commitment problems," 1993 IEEE International Symposium on Circuits and Systems, Chicago, IL, vol.4, 1993, pp. 2204-2207, doi: 10.1109/ISCAS.1993.394197.

[2] Wood A. J., B. F. Wollenberg, "Power Generation, Operation, and Control," New York: Wiley, 1996.

[3] T. Li and M. Shahidehpour, "Price-based unit commitment: A case of Lagrangian relaxation versus mixed integer programming," IEEE Trans. on Power Systems, vol. 20, no. 4, pp. 2015-2025, 2005.

[4] F. Yu, F. Tu, H. Tu and K. R. Pattipati, "A Lagrangian Relaxation Algorithm for Finding the MAP Configuration in QMR-DT," in IEEE Transactions on Systems, Man, and Cybernetics - Part A: Systems and Humans, vol. 37, no. 5, pp. 746-757, Sept. 2007, doi: 10.1109/TSMCA.2007.902657.

[5] A. K. Zadeh, K. M. Nor and H. Zeynal, "Multi-thread security constraint economic dispatch with exact loss formulation," 2010 IEEE International Conference on Power and Energy, Kuala Lumpur, 2010, pp. 864-869, doi: 10.1109/PECON.2010.5697700.

[6] M. Eidiani, "A reliable and efficient method for assessing voltage stability in transmission and distribution networks," International Journal of Electrical Power Energy Systems, vol. 33, no. 3, pp. 453-6, 2011.

[7] Ostrowski J., M. F. Anjos, and A. Vannelli, "Tight Mixed Integer Linear Programming Formulations for the Unit Commitment Problem," IEEE Trans. on Power Systems, vol. 27, no. 1, pp. 39-46, 2012, doi: 10.1109/TPWRS.2011.2162008.

[8] W. Shouxiang, Z. Na, L. Zuyi, and M. Shahidehpour, "Modeling and impact analysis of large scale V2G electric vehicles on the power grid," in Innovative Smart Grid Technologies - Asia (ISGT Asia), 2012 IEEE, 2012, pp. 1-6, doi: 10.1109/ISGT-Asia.2012.6303372.

[9] M. E. Khodayar, W. Lei, and L. Zuyi, "Electric Vehicle Mobility in Transmission-Constrained Hourly Power Generation Scheduling," Smart Grid, IEEE Transactions on, vol. 4, no. 2, pp. 779-788, 2013, doi: 10.1109/TSG.2012.2230345. 
[10] Q. Jiang, B. Zhou and M. Zhang, "Parallel augment Lagrangian relaxation method for transient stability constrained unit commitment," in IEEE Transactions on Power Systems, vol. 28, no. 2, pp. 1140-1148, May 2013, doi: 10.1109/TPWRS.2012.2216553.

[11] H. Zeynal, M. Eidiani, "Hydrothermal Scheduling Flexibility Enhancement with Pumped-Storage Units," 22nd Iranian IEEE Conference on Electrical Engineering (ICEE), Tehran, Iran, 2014, pp. 820-825.

[12] H. Zeynal, L. X. Hui, Y. Jiazhen, M. Eidiani and B. Azzopardi, "Improving Lagrangian Relaxation Unit Commitment with Cuckoo Search Algorithm," 2014 IEEE International Conference on Power and Energy (PECon), Kuching, 2014, pp. 77-82, doi: 10.1109/PECON.2014.7062417.

[13] H. Zeynal, Y. Jiazhen, B. Azzopardi and M. Eidiani, "Flexible economic load dispatch integrating electric vehicles," 2014 IEEE 8th International Power Engineering and Optimization Conference (PEOCO2014), Langkawi, 2014, pp. 520-525, doi: 10.1109/PEOCO.2014.6814484.

[14] H. Zeynal, Y. Jiazhen, B. Azzopardi and M. Eidiani, "Impact of Electric Vehicle's integration into the economic VAr dispatch algorithm," 2014 IEEE Innovative Smart Grid Technologies - Asia (ISGT ASIA), Kuala Lumpur, 2014, pp. 780-785, doi: 10.1109/ISGT-Asia.2014.6873892.

[15] N. Karthik, A.K. Parvathy, R. Arul, "Non-convex Economic Load Dispatch using Cuckoo Search Algorithm," Indonesian Journal of Electrical Engineering and Computer Science, vol. 5, no 1, pp. 48-57, January 2017, doi: 10.11591/ijeecs.v5.i1.pp48-57.

[16] J. Zhao, S. Liu, M. Zhou, X. Guo and L. Qi, "An Improved Binary Cuckoo Search Algorithm for Solving Unit Commitment Problems: Methodological Description," in IEEE Access, vol. 6, pp. 43535-43545, 2018, doi: 10.1109/ACCESS.2018.2861319.

[17] K. Kim, A. Botterud and F. Qiu, "Temporal Decomposition for Improved Unit Commitment in Power System Production Cost Modeling," in IEEE Transactions on Power Systems, vol. 33, no. 5, pp. 5276-5287, Sept. 2018, doi: 10.1109/TPWRS.2018.2816463.

[18] X. Sun, P. B. Luh, M. A. Bragin, Y. Chen, J. Wan and F. Wang, "A Novel Decomposition and Coordination Approach for Large Day-Ahead Unit Commitment with Combined Cycle Units," in IEEE Transactions on Power Systems, vol. 33, no. 5, pp. 5297-5308, Sept. 2018, doi: 10.1109/TPWRS.2018.2808272.

[19] Z. M. Yasin, N. F. A. Aziz, N. A. Salim, N. A. Wahab, N. A. Rahmat, "Optimal Economic Load Dispatch using Multiobjective Cuckoo Search Algorithm,” Indonesian Journal of Electrical Engineering and Computer Science, vol.2, no. 1, pp. 168-174, Oct 2018, doi: 10.11591/ijeecs.v12.i1.pp168-174.

[20] A. M. Palani, H. Wu and M. M. Morcos, "A Frank-Wolfe Progressive Hedging Algorithm for Improved Lower Bounds in Stochastic SCUC," in IEEE Access, vol. 7, pp. 99398-99406, 2019. doi: 10.1109/ACCESS.2019.2927346.

[21] T. Ahmed Khan, M. J. Tahir, M. Alam, K. A. Kadir, Z. Shahid, "Optimized health parameters using PSO: a cost effective rfid based wearable gadget with less false alarm rate," Indonesian Journal of Electrical Engineering and Computer Science, vol. 15, no 1, pp. 230-239, July 2019, doi: 0.11591/ijeecs.v15.i1.pp230-239.

[22] J. Shen, L. Hu, C. Cheng and S. Wang, "Automatic generation control of a large hydropower plant with head-sensitive forbidden and restricted zones," in IET Renewable Power Generation, vol. 14, no. 7, pp. 1113-1123, 2020, doi: 10.1049/iet-rpg.2019.1055.

[23] X. Li, Q. Zhai, J. Zhou and X. Guan, "A Variable Reduction Method for Large-Scale Unit Commitment," in IEEE Transactions on Power Systems, vol. 35, no. 1, pp. 261-272, Jan. 2020, doi: 10.1109/TPWRS.2019.2930571.

[24] Rana Z. Alkawaz, Abbas Y. AlBayati, Marwan S. Jameel," Interaction between updated FR-CG algorithms with optimal Cuckoo algorithm," Indonesian Journal of Electrical Engineering and Computer Science, vol 19, no 3, pp.1497-1504, Sept 2020, doi: 10.11591/ijeecs.v19.i3.pp1497-1504.

[25] Y. Xin-She and S. Deb, "Cuckoo Search via Lévy flights," in Nature \& Biologically Inspired Computing," 2009, NaBIC 2009. World Congress on, pp. 210-214, 2009.

[26] X. S. Yang, S. Deb, "Engineering optimization by cuckoo search," Int. J. Mathematical Modelling and Numerical Optimization, vol. 1, no. 4, pp. 330-343, 2010, doi: 10.1504/IJMMNO.2010.035430.

[27] R. Rajabioun, "Cuckoo Optimization Algorithm," Applied Soft Computing, vol. 11, no. 8, pp. 5508-5518, 2011, doi: 10.1016/j.asoc.2011.05.008. 